ORIGINAL ARTICLE

\title{
To lead or not to lead? Prospective controlled study of emergency nurses' provision of advanced life support team leadership
}

\author{
P Gilligan, C Bhatarcharjee, G Knight, M Smith, D Hegarty, A Shenton, F Todd, P Bradley
}

Emerg Med J 2005;22:628-632. doi: 10.1136/emj.2004.015321

See end of article for authors' affiliations

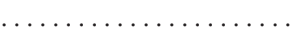

Correspondence to: P Gilligan, Specialist Registrar in Emergency Medicine on the Yorkshire Rotation, UK hegartydeirdre@ireland. com

Accepted for publication 25 September 2004
Background and objectives: In many emergency departments advanced life support (ALS) trained nurses do not assume a lead role in advanced resuscitation. This study investigated whether emergency nurses with previous ALS training provided good team leadership in a simulated cardiac arrest situation.

Methods: A prospective study was conducted at five emergency departments and one nurses' association meeting. All participants went through the same scenario. Details recorded included baseline blood pressure and pulse rate, time in post, time of ALS training, and subjective stress score (1 = hardly stressed; $10=$ extremely stressed). Scoring took into account scenario understanding, rhythm recognition, time to defibrillation, appropriateness of interventions, and theoretical knowledge.

Results: Of 57 participants, 20 were ALS trained nurses, 19 were ALS trained emergency senior house officers (SHOs), and 18 were emergency SHOs without formal ALS training. The overall mean score for doctors without ALS training was 69.5\%, compared with 72.3\% for ALS trained doctors and 73.7\% for ALS trained nurses. Nurses found the experience less stressful (subjective stress score 5.78/10) compared with doctors without ALS training (6.5/10). The mean time taken to defibrillate from the appearance of a shockable rhythm on the monitor by the nurses and those SHOs without ALS training was 42 and 40.8 seconds, respectively.

Conclusion: ALS trained nurses performed as well as ALS trained and non ALS trained emergency SHOs in a simulated cardiac arrest situation and had greater awareness of the potentially reversible causes of cardiac arrest. Thus if a senior or middle grade doctor is not available to lead the resuscitation team, it may be appropriate for experienced nursing staff with ALS training to act as ALS team leaders rather than SHOs.
A paradox seems to exist in that many nurses act as instructors on resuscitation training courses but do not in reality act as resuscitation team leaders. This would seem to imply that nurses are good enough to teach advanced life support but not to lead teams involved in it. As noted by O'Higgins et al: "Acquiring ALS provider status is not necessarily a licence to practice and individual hospital policy determines which skills these nurses can perform without direct medical supervision." ${ }^{11}$ Their survey demonstrated that many acute hospitals do not permit nurse advanced life support (ALS) providers to use a number of skills taught on the ALS provider course. In 1993, the Advanced Life Support Group recommended that: "one member must assume the role of team leader and, if only one person was a doctor or an advanced cardiac life support provider, the choice of leader should be the life support provider."2

The patients most likely to survive in-hospital cardiac arrests are those in whom return of spontaneous circulation has been achieved by the first responder. In most clinical areas the first responder is likely to be a nurse. ${ }^{34}$ In their questionnaire based study of 112 qualified nurses, Stewart and Lowe found that $75 \%$ had been involved in cardiopulmonary resuscitation (CPR) as first responders. ${ }^{5}$ Jones and Cooke, in their paper on the role of the rural registered nurse in advanced life support, remarked: "Often nurses voice their frustrations at being in a situation where they know what must be done but have to wait for the arrival of a medical officer to initiate known actions."

A search of the literature revealed that although a number of papers have examined the attitudes of nurses and doctors to ALS team leadership, few papers have reported data regarding prospective controlled trials on the subject. ${ }^{78}$ The importance of training in ALS is apparent from Dane et al's study into the association between Advanced Cardiac Life Support (ACLS; the ALS course is similar in content and delivery to the ACLS) training and survival of patients after in-hospital cardiac arrest. In their study they found that patients who arrested and were discovered by an ACLS trained nurse were about four times more likely to survive than were patients discovered by a nurse, without training in ACLS. ${ }^{9}$

This study was undertaken to test our hypothesis that ALS trained accident and emergency (A\&E) nurses would perform as well as A\&E senior house officers (SHOs) both with and without ALS training in the role of team leader in a simulated cardiac arrest scenario.

\section{METHODS}

We obtained prior permission to attend the emergency departments of five hospitals in the Yorkshire region of the UK from senior medical and nursing staff at these institutions. The research team (consisting of a resuscitation training officer, an ALS trained doctor and an assistant) went to each department and set up to run the simulated scenario.

The scenario involved a distressed 45 year old man (a mannequin lying on the bed) brought to hospital with severe chest pain, dyspnoea, and nausea. He had no significant past medical history and was on no medication. The patient was

Abbreviations: A\&E, accident and emergency; ALS, advanced life support; SHO, senior house officer 
described as being pale and sweaty. He was said to have a pulse of 110 beats per minute and to be hypertensive with a blood pressure of $150 / 90 \mathrm{~mm} \mathrm{Hg}$. One minute into the scenario the patient collapsed and was pulseless, the monitor revealed ventricular tachycardia that quickly degenerated to ventricular fibrillation. The rhythm changed to pulseless electrical activity/electromechanical dissociation after two cycles of stacked shocks initially at $200 \mathrm{~J}, 200 \mathrm{~J}$, and $360 \mathrm{~J}$ with a minute of CPR and adrenaline and a further three shocks at $360 \mathrm{~J}$. At this point the participant (nurse or SHO) was asked about the possible causes of a cardiac arrest. If the mannequin had not been intubated, the member of the research team running the scenario pointed out that the patient was cyanosed. Intubation and continued life support would result in a change on the monitor to sinus tachycardia with the return of a palpable pulse after one minute. The participant was then asked what investigations should be arranged, and they were expected to arrange the appropriate referral. The research team felt such a scenario would assess the important skills and knowledge required to lead an ALS attempt because it addressed most of the potential rhythms, their recognition, and the appropriate interventions.

The members of staff of the emergency department (ALS trained nurses, ALS trained doctors and non ALS trained doctors) were then approached individually and asked to volunteer to act as team leader for a simulated cardiac arrest. The study was performed using a resuscitation mannequin and the research team provided the members of the resuscitation team. The scenario was dictated to the team leader (that is, the participant) by the lead member of the research team who read it verbatim from a preprinted sheet. All participants experienced the same scenario. The resuscitation team members offered no clues-verbal or otherwiseto the team leader, that is, the team members did not speak during the test except to introduce themselves and answer questions posed by the team leader.

On agreeing to take part the participants were brought to the designated area, their pulse and blood pressure was recorded, and then the scenario was described. Their performance was scored according to a predetermined scoring system (box 1) based on scenario understanding, team orientation to tasks, preparation, rhythm recognition, sequencing of interventions, appropriateness of interventions, and performance of defibrillation.

The research team recorded the results in the form of numerical scores. Data from the scoring sheets were entered onto a Microsoft Excel spreadsheet. We analysed the data using Stata Corp., 2001. Stata Statistical Software: Release 7.0, College Station. When departures from normality of the scores were not marked we used analysis of variance (ANOVA) to make comparisons across the three groups. Where the scores in one or more groups departed grossly from a normal distribution the equivalent non-parametric test (Kruskal-Wallis test) was performed.

\section{RESULTS}

We tested a total of 57 participants. Of these, 20 were ALS trained nurses, 19 were ALS trained A\&E SHOs, and 18 were A\&E SHOs without formal ALS training. It is worth noting all of the SHOs in the study would have received informal inhouse training in ALS as part of their induction programme but only those who had undergone a Resuscitation Council (UK) recognised course were deemed to be ALS trained. The mean post-qualification time for nurses was about 10 years and for doctors with ALS training this was over two and a half years, whereas it was on average two years for the doctors without ALS training. The results of the three groups are shown in table 1.

\section{Box 1: Scoring sheet}

- Candidate number

- Profession

- ALS provider status

- Date of ALS course

- Years post graduation

- Time working in A\&E

- Pre-test BP and pulse

- Post-test BP and pulse

Score ( 1 if performed; 0 if not)

- Orients team to task

- Assesses airway

- Assesses breathing

- Assesses circulation

- Orders high flow oxygen

- Recognises sinus tachycardia

- Recognises ventricular fibrillation

- Time taken to recognise defibrillation

- Places gel pads

- Charges paddles on patient's chest

- Orders team to stand clear

- Delivers shock at 200 joules

- Delivers shock at 200 joules

- Delivers shock at 360 joules

- Orders intubation of the patient

- Orders appropriate checks of tube

- Time to delivery of first shock

- Orders CPR for 1 minute

- Orders adrenaline

- Delivers shock at 360 joules

- Delivers shock at 360 joules

- Delivers shock at 360 joules

- Recognises sinus rhythm on monitor

- Performs pulse check

- Recognises pulseless electrical activity

- Indicates 8 possible reversible causes, 1 point being awarded for each

- Recognises change to sinus tachycardia

- Rechecks pulse and blood pressure

- Orders full blood count, blood gas, cardiac enzymes, urea and electrolytes, glucose, chest $x$ ray, 1 point each

- Refers to CCU/ITU

- Participant's post test subjective stress score on a scale of 1 to 10

The nurses had worked for a mean (SD) of 82.4 (47) months (range 18-204) in A\&E, the doctors with ALS training for a mean of 6.6 (6.3) months (range 1-24), and the doctors without formal ALS training for a mean of 5.2 (2.88) months (range 1-12).

\section{Subjective stress score}

We felt this was an important outcome variable. If one group found it more stressful than the other this might be an indication not to expect them to lead the team. The score was obtained by asking the participants how stressful they had found it as soon as they completed the scenario. 


\begin{tabular}{|c|c|c|c|}
\hline & ALS trained nurses & ALS trained doctors & $\begin{array}{l}\text { Doctors without } \\
\text { formal ALS training }\end{array}$ \\
\hline Time post qualification (months) & $\begin{array}{l}121.7(83.60) \\
30-312\end{array}$ & $\begin{array}{l}31.75(27.2) ; \\
6-132\end{array}$ & $\begin{array}{l}24(7.9) ; \\
12-42\end{array}$ \\
\hline $\begin{array}{l}\text { Time to recognition of ventricular } \\
\text { fibrillation (seconds) }\end{array}$ & $\begin{array}{l}12.4(16.5) \\
3-65\end{array}$ & $\begin{array}{l}13.2(16.3) ; \\
1-56\end{array}$ & $\begin{array}{l}10(9.5) \\
2-32\end{array}$ \\
\hline $\begin{array}{l}\text { Time taken to defibrillate from the } \\
\text { appearance of ventricular fibrillation } \\
\text { on the monitor (seconds) }\end{array}$ & $\begin{array}{l}42(44.6) ; \\
11-200\end{array}$ & $\begin{array}{l}36.8(28.7) ; \\
9-109\end{array}$ & $\begin{array}{l}40.8(19.9) \\
15-85\end{array}$ \\
\hline Knowledge of the eight reversible causes & $\begin{array}{l}6.71(1.98) ; \\
0-8\end{array}$ & $\begin{array}{l}6.9(0.85) \\
5-8\end{array}$ & $\begin{array}{l}5.6(1.37) \\
3-8\end{array}$ \\
\hline $\begin{array}{l}\text { Subjective stress score }(1=\text { hardly } \\
\text { stressed; } 10=\text { extremely stressed })\end{array}$ & $\begin{array}{l}5.78(2.3) \\
2-10\end{array}$ & $\begin{array}{l}6.4(1.57) \\
3-8.5\end{array}$ & $\begin{array}{l}6.5(1.9) ; \\
3-9\end{array}$ \\
\hline Total score out of a possible 40 points & $\begin{array}{l}29.48(5.77) ; \\
7-36\end{array}$ & $\begin{array}{l}28.9(3.26) ; \\
21-33\end{array}$ & $\begin{array}{l}27.8(4.78) \\
14-34\end{array}$ \\
\hline
\end{tabular}

They were asked to score themselves on a scale of 1-10 where $1=$ hardly stressed at all and $10=$ extremely stressed. The mean scores were: nurses 5.78 (2.3), doctors with ALS 6.4 (1.57), and doctors without ALS 6.5 (1.9). No significant difference was found between the groups (ANOVA; $\mathrm{p}=0.49$ ).

\section{Alteration in pulse rate}

These data were gathered as a crude marker of physiological stress as was the alteration in mean arterial pressure (MAP). The change in pulse rate was recorded for each group by subtracting the pretest pulse rate from the post-test pulse rate. No significant difference was found between the three groups (ANOVA; $\mathrm{p}=0.9$ ).

\section{Alteration in MAP}

The change in MAP for each participant was calculated by checking the blood pressure before and immediately after the scenario. Subtracting the pretest from the post-test reading gave us the alteration in MAP. No significant difference was found between the groups (ANOVA; $\mathrm{p}=0.95$ ).

It would appear from these data that the stress levels and change in crude physiological markers of stress for doctors and nurses were similar.

\section{Time taken to defibrillate}

This outcome measure is important, as we know that the sooner patients are defibrillated from a shockable rhythm the more likely they are to survive. If there was an appreciable delay between the groups this would be of clinical significance. Time taken to defibrillate was calculated from appearance of a shockable rhythm on the monitor until the first shock was delivered. The nurses took a mean of 42 (44.6) seconds, ALS trained doctors took 36.8 (28.7) seconds, and doctors without formal ALS training took 40.8 (19.9) seconds (table 1). The difference was not clinically or statistically significant $(\mathrm{p}=0.95)$.

Knowledge of the eight potentially reversible causes The Resuscitation Council (UK) suggests that there are eight potentially reversible causes of cardiac arrest that should be considered and if confirmed treated..$^{10}$ We asked our participants for the possible causes and scores were given out of a possible 8 . The nurses scored a mean of 6.71 (1.98), the doctors with ALS training 6.9 (0.85), and the doctors without formal ALS training 5.6 (1.37). Of note is that the nurses had a median score equal to the maximum, that is, half of the nurses knew all of the possible causes whereas this was true for only a quarter of the doctors without ALS training. This difference reached statistical significance (Kruskal-Wallis Test; $\left.\chi^{2}=9.95, \mathrm{df}=2, \mathrm{p}=0.007\right)$. This is probably of clinical significance because if the team leader does not know the potential causes these may not be discovered and treated.

\section{Total score}

The maximum possible score for the scenario was 40 . The nurses scored a mean of 29.48 (5.77), doctors with ALS 28.9 (3.26) and doctors without formal ALS training 27.8 (4.78). The differences failed to reach statistical significance (ANOVA; $\mathrm{p}=0.54$ ).

\section{DISCUSSION}

The Resuscitation Council takes a multiprofessional approach to ALS education. Harden advised that: "when planning multi-professional education one has to choose an approach appropriate to the goals and context." ${ }^{\prime 11}$ This is certainly the case with regards to resuscitation training as it is appropriate that all members of the team know the theory that underlies the practice and are proficient in the skills required. Furthermore, Pirrie et al in their study on multiprofessional education noted: "There was a widespread recognition that no single profession had a monopoly of the knowledge base required to deliver efficient patient care."12

By empowering A\&E nurses to assume a leadership role in ALS we may facilitate earlier provision of basic life support and defibrillation so saving more lives. We were interested to find that neither the nurses nor the doctors found the procedure significantly more stressful than the other group. This was corroborated by crude physiological assessments of stress in the form of altered blood pressure and pulse. This implies that neither found the leadership role more psychologically or physiologically challenging than the other, and this should not deter those nurses with the necessary skills from adopting the leadership role.

We used a simulated cardiac arrest scenario to assess the team leadership performance of our participants. Kaye and Mancini found this allowed for effective evaluation of individual and group performance in their study. ${ }^{13}$ They compared 32 medical residents with nine critical care registered nurses and found that the doctors, although poor, were better in the assessment of patient status than the nurses. However, with regard to drug therapy and problem solving no difference was found. ${ }^{13}$ We did not find any statistically significant differences in the present study between the performance of ALS trained and non ALS trained A\&E SHOs when compared with ALS trained A\&E nurses, except in the area of knowledge of aetiology in which the nurses performed better. 
Although we assessed the performance of the team leader (the participant) in terms of adherence to ALS protocols we did not assess other aspects of leadership, for example degree of assertiveness, voice projection, team confidence in the leader or other parameters that might be regarded as intrinsic to the role of the leader. Although ALS training may improve performance as a team leader we accept that it does not imply competence in this role. Cooper and Wakelman, who conducted an observational study of team leadership in 20 resuscitation attempts, concluded their report by remarking that: "The key findings indicate that there is an effective way to lead an emergency team, namely through the initiation of structure and establishing a system of control that directs, guides, co-ordinates and maintains performance standards." ${ }^{14}$ As these are the central components of ALS training, and as the time to provision of basic life support and defibrillation is the key to improved survival we feel our approach and methods are valid.

The recruitment could not be formally randomised but it did depend on who was available in the department when we arrived, except in the context of the Yorkshire A\&E Nurses Association Meeting. We accept that the research team was aware of the profession of the participant and their ALS provider status and that this knowledge had the potential to bias our results. We did, however, follow a strict marking system which involved a single mark for the correct response and no mark for any omission. The researcher running the scenario did so from a preprinted sheet that clearly set out the information to be given to the participants and thus we hope the lack of blinding has not biased our findings.

The fact that nurses more frequently declined to be involved in the study than doctors may have affected our results, but this also reflects the reality that many nurses might not wish to assume the role of ALS team leader and so we feel the comparison remains valid. The nurses group was significantly more experienced than the two SHO groups and some may regard the comparison as unfair. This again reflects the reality of emergency departments. The more senior nurses tend to be the ones who are ALS trained as funding for sending nurses on such courses is limited.

We found no significant difference in the overall performance between the three groups of participants, two of which had had ALS training, although the nurses had a statistically significant greater knowledge of the potential causes of cardiac arrest. It is again worth noting that the A\&E SHOs all had informal training in ALS as part of their induction training and in their ongoing educational programme. This may explain the lack of statistical difference between the performance of the three groups. Nurses without ALS training were not included in the study as we felt it would be difficult to recruit for this group and it would not be relevant as nurses without ALS training would be unlikely to want to assume the role of ALS team leader.

This study did not look at doctors or nurses working on wards or outside the setting of A\&E departments. Given that $A \& E$ nurses and doctors are more frequently exposed to cardiac arrests and that A\&E SHOs are generally taught resuscitation skills during the induction programme we accept that the results may not apply to other hospital settings.

Some of the nursing staff who volunteered for the study were ALS instructors and this might have influenced some of the results. We do not feel that this detracts from the study as in reality some A\&E nurses are ALS instructors and it would have been wrong to exclude such nurses from a comparative study. Local hospital policy still dictates whether or not such nurses act as team leaders for resuscitation efforts.
Senior and middle grade doctors were not included in the study. Until recently many departments did not have 24 hour middle grade shop floor presence. If a senior or middle grade doctor is available they may reasonably be expected to lead a resuscitation effort. However, the choice of who should lead between an experienced ALS trained nurse or an SHO is less obvious-hence the relevance of this study.

\section{CONCLUSION}

Our study has shown that A\&E nurses with ALS training perform as well as A\&E SHOs with or without ALS training in a simulated cardiac arrest. They find it no more stressful than the SHOs and they defibrillate equally rapidly. In some cases, the nurses have greater knowledge of the potentially reversible causes. The Secretary of State in the introduction to the NHS Plan (2000) advises "For the first time, nurses and other health professionals will be given the bigger roles that their qualifications and expertise deserve." ${ }^{15}$ Those involved in emergency medicine may need to consider empowering experienced nurses to use the knowledge and skills they acquire from ALS training to assume the role of ALS team leaders where a senior doctor is not available.

\section{AUTHORS' CONTRIBUTIONS}

All the authors were involved in the design of the study. The first three authors were the research team. Dr Gilligan drafted the paper and all of the authors contributed to the editing.

\section{ACKNOWLEDGEMENTS}

We would like to thank the staff of the accident and emergency departments of the Bradford Royal Infirmary, Airedale Hospital, Dewsbury District General Hospital, Leeds General Infirmary, and St James's University Hospital for their participation in this study.

\section{Authors' affiliations \\ P Gilligan, G Knight, A Shenton, F Todd, P Bradley, Bradford Royal \\ Infirmary, Bradford, UK \\ C Bhatarchariee, Family Practitioner, Bradford, UK \\ M Smith, Family Practitioner, Bradford, UK \\ D Hegarty, Family Practitioner, Leeds, UK}

This research was funded by the Accident and Emergency Department of the Bradford Royal Infirmary, Bradford, UK. All of the equipment required in the study was provided by the Resuscitation Training Department of the Bradford Royal Infirmary.

Competing interests: none declared

We approached the ethics committee chair of the Bradford Royal infirmary in 2000 and he was of the opinion that the scenario based study did not require formal approval at that time and that we could proceed.

\section{REFERENCES}

1 O'Higgins F, Ward M, Nolan J. Advanced life support skills undertaken by nurses-UK survey. Resuscitation 2001:50:45-9.

2 Advanced Life Support Group. Cardiac life support-The practical approach. London: Chapman and Hall Medical, 1993.

3 Coady EM. A strategy for nurse defibrillation in general wards. Resuscitation 1999;42:183-6.

4 Finn J. The role of nurses in cardiopulmonary resuscitation and defibrillation. Collegian 1996;3:31-4

5 Stewart AJ, Lowe MD. Knowledge and attitude of nurses on medical wards to defibrillation. JR Coll Phys Lond 1994;28:399-401.

6 Jones J, Cooke T. Advanced life support: Role of the rural registered nurse. Aust J Rural Health 1996;4:67-72.

7 Swanson RW, Ramsden VR. Advanced cardiac life support: A survey of interprofessional attitudes. Heart Lung 1988;17:254-5.

8 Gibson S. The nurse as team leader during advanced life support in A\&E. Emerg Nurse 1998;6:30-2.

9 Dane FC, Russell-Lindgren KS, Parish DD, et al. In-hospital resuscitation: Association between ACLS training and survival to discharge. Resuscitation 2000;47:83-7.

10 Advanced Life Support Course Provider Manual, 4th edn. London: Resuscitation Council (UK), 2000. 
11 Harden RM. AMEE Guide No. 12: Multiprofessional education, Part 1Effective multiprofessional education: a three-dimensional perspective. Med Teach 1998;20:402-8.

12 Pirrie A, Wilson V, Harden RM, et al. AMEE Guide No. 12: Multiprofessional education, Part 2-Promoting cohesive practice in health care. Med Teach 1998;20:409-15.
13 Kaye W. Mancini M. Use of the mega code to evaluate team leader performance during advanced cardiac life support. Crit Care Med 1986;14:99-104. 14 Cooper S, Wakelman A. Leadership of resuscitation teams: "Lighthouse Leadership". Resuscitation 1999:42:27-45.

15 Department of Health. The NHS Plan: a plan for investment, a plan for reform. London: $\mathrm{DoH}, 2000$. 